\section{Feasibility of bimanual microincision phacoemulsification in hard cataracts}

A Assaf and AM El-Moatassem Kotb

\begin{abstract}
Purpose The purpose of this work was to assess the feasibility of bimanual phacoemulsification in hard cataracts of N3+ using WhiteStar technology of Soveriegn (Advanced Medical Optics, Santa Ana, CA, USA).

Setting Ain-Shams University Hospitals, Ophthalmology Department, Cairo, Egypt.

Design A randomized prospective noncomparative study.
\end{abstract}

Methods A randomized prospective study of 33 consecutive cases ( $\mathrm{N} 3+$ or more) was conducted, phacoemulsification using a bimanual microincision technique using the Sovereign with WhiteStar technology phacoemulsification machine. One phaco mode was used in all eyes. The ultrasound power was set at $30-25 \%$ according to the hardness of the nuclei, duty cycle of $33 \%$, flow rate of $20-28 \mathrm{~cm}^{3} / \mathrm{min}$, and vacuum of $240 \mathrm{mmHg}$. Occlusion mode was on. Nine eyes received rollable intraocular lenses (IOL) of ThinOptx, whereas 24 eyes had been implanted with hydrophobic acrylic foldable IOL (Sensar OptiEdge SA40e of AMO) through a third incision. Study parameters were effective phacotime (EPT), presence of wound burn, degree of immediate postoperative iritis, amount of infusion solution used, and total operating time. Results The mean EPT was $4.3 \mathrm{~s}$ with an average ultrasound used of $5.7 \%$.

The mean operating time was $11 \mathrm{~min}$ and $20 \mathrm{~s}$. Although the nuclear hardness was of grade 3 or above (in a scale of 5), there were no cases of thermal burn; $P=0.005$. Only three eyes suffered postoperative iritis $2+$, which resolved within 1 week on topical steroids, statistically nonsignificant, $P=0.2$. The amount of infusion solution was less than that used in conventional coaxial phaco. This technique induced considerably less corneal astigmatism than surgery using conventional corneal incisions.

Conclusions Hard cataracts of N3 or more could be safely removed through an incision of $1.4 \mathrm{~mm}$ incision using bimanual micro-phaco.

Eye (2007) 21, 807-811; doi:10.1038/sj.eye.6702356; published online 5 May 2006

Keywords: bimanual phacoemulsification; microincision phacoemulsification; hard cataracts; rollable IOL; effective phacotime (EPT); MICS

\section{Introduction}

The advent of phacoemulsification in the late 1960s and extracapsular cataract extraction led to improved surgical outcomes, fewer postoperative infections, and faster operations with improved visual outcomes as a result of using smaller incisions and leaving the posterior capsule intact. ${ }^{1}$ The next major step for cataract surgery may be microincisional bimanual phacoemulsification. Bimanual phacoemulsification through a $1 \mathrm{~mm}$ incision was first described by Shearing et $a l^{2}$ in 1985. However, because intraocular lenses (IOL) at the time required incisions of 5.5-6.0 mm, interest in bimanual phacoemulsification was limited. ${ }^{3}$ In the last 6 years, a number of groups have shown renewed interest in bimanual phacoemulsification and are studying new techniques and technologies to improve the safety of bimanual phacoemulsification.

Bimanual phacoemulsification is different from traditional phacoemulsification because the irrigating and aspirating functions of the phaco handpiece are separated into two different handpieces. As a result, one
Department of Ophthalmology, Ain-Shams University, Nasr City, Cairo, Egypt

Correspondence: AM El-Moatassem Kotb, Department of Ophthalmology, Ain-Shams University, 46 Dr Ahmed Mohamed Ibrahim St., Nasr City, Cairo 11471, Egypt

Tel: + 202287 3098;

Fax: + 2022873498

E-mail: ahmedelmotasem@ yahoo.com

Received: 5 July 2005 Accepted in revised form: 13 March 2006 Published online: 5 May 2006

None of the authors has a financial or proprietary interest in any of the products mentioned 
instrument provides irrigation to the anterior chamber, and the other instrument phacoemulsifies and aspirates the nucleus. Because irrigation is no longer provided through the phaco sleeve, a bare needle can be used to emulsify the nuclear fragments. The phaco needle is of small diameter, and incisions of less than $1.5 \mathrm{~mm}$ are typically used to insert the needle into the anterior chamber.

Continual improvements in phacoemulsification technique and technology have made cataract surgery more safe and efficient than was possible in the past. In general, the major trends in phacoemulsification have been the use of smaller incisions, improved fluidics using higher levels of vacuum for lens extraction with less dependence on ultrasound energy for emulsification, and increasing use of alternative energy sources and adjunctive devices during surgery. The quest for lens extraction with reduced intraocular energy has been accomplished with power modulations and supplemental energy sources, with the result of improved outcomes. ${ }^{4,5}$

\section{Purpose of the work}

This study was aimed to assess the feasibility of performing phacoemulsification through microincisions of $1.4 \mathrm{~mm}$ as well as to assess presence of any relative intraoperative and postoperative complications.

\section{Participants and methods}

\section{The surgical procedure}

\section{Machine setting}

One phaco mode was used in all eyes. The ultrasound power was set at 30-25\% according to the hardness of the nuclei. WhiteStar was kept on during all phases of the surgery, with duty cycle of $33 \%$, flow rate of $20-28 \mathrm{~cm}^{3} / \mathrm{min}$, and vacuum of $240 \mathrm{mmHg}$.

Study parameters were EPT (effective phaco time), presence of wound burn, degree of immediate postoperative iritis, amount of infusion solution used, and total operating time.

\section{Technique}

Corneal incision Two $1.4 \mathrm{~mm}$ stab incisions were created in the mid-limbal zone approximately at $10 \mathrm{O}^{\prime}$ clock and $2 \mathrm{O}^{\prime}$ clock positions. The outer lip of the incision was made slightly larger than inlet (trapezoid) to facilitate instrument introduction and manipulation through it.

Capsulorrhexis Viscoelastic was injected into the anterior chamber. The capsulorrhexis was made by a $24-\mathrm{G}$ needle, followed by aspiration of the capsule out of the anterior chamber by using a 21 cannula attached to $10 \mathrm{~cm}^{3}$ syringe. Hydrodissection was then performed followed by hydrodelineation.

Bimanual phacoemulsification A 20-G irrigating chopper (Neumann-type chopper with two side irrigating ports) was inserted first through one of the stap incisions followed by insertion of the micro-phaco needle $(0.9 \mathrm{~mm})$.

The technique utilized for all cases was Quick Chop (Phaco Chop). After activation of high vacuum, the phaco needle was impaled within the centre of the nucleus. This was followed by chopping of the nucleus vertically into multiple pieces (four or more). Free nuclear fragments were then retrieved and emulsified in an endocapsular, or in a supracapsular plane in cases of shallow anterior chamber owing to large lens (Figure 1).

Irrigation and aspiration of cortex The 20-G bimanual irrigating and aspirating instruments were each inserted into the eye. The aspirating probe was used to remove cortex, and if there were difficulty removing the subincisional cortex, the probes might be switched to reach the subincisional cortex.

Intraocular lens insertion By completion of phaco procedure, nine eyes received rollable lens (Ultra Choice 1.0), IOL of ThinOptx, whereas in 24 eyes a $3.0 \mathrm{~mm}$ third incision was created for implantation of hydrophobic acrylic IOL (Sensar AR40e IOL of AMO). After the injection of the IOL into the capsular bag, residual viscoelasticity was removed (bicorneal, corneal), and the two stat incisions were stromally hydrated; the third incision was found clean unmanipulated, and needed no stromal hydration in all cases.

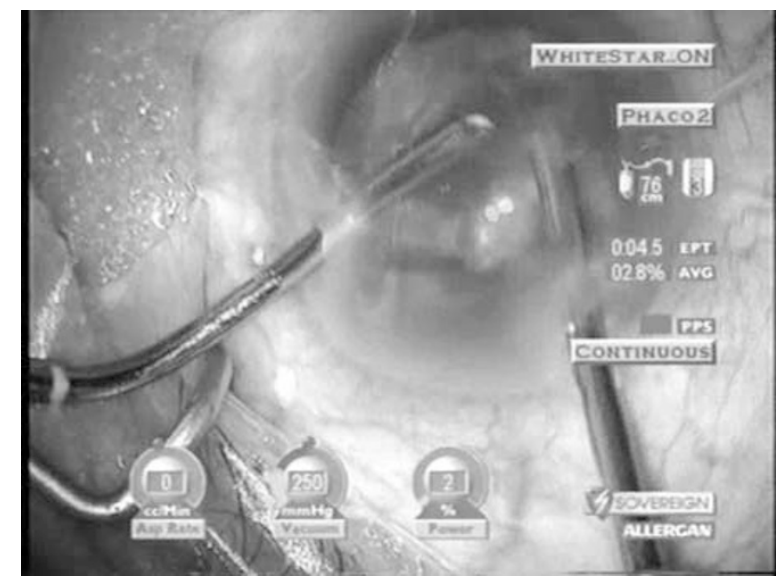

Figure 1 Bimanual phaco. 


\section{Data analysis}

Data were collected postoperatively and entered into an Excel spreadsheet for subsequent analysis (Microsoft Inc., Seattle, WA, USA). Data are reported as mean \pm s.d. Statistical significance was determined using Student's $t$-test for associations of patient age and refractive error with retreatment incidence. A $P$-value of 0.05 was considered statistically significant.

\section{Results}

Thirty-three patients ( 25 men, 75\% and eight women, 25\%; age range between 55 and 73 years) participated in

Table 1 Average parameters used

\begin{tabular}{lcl}
\hline Effective phaco time (min) & $4.3 \mathrm{~s}$ & $P=0.005^{*}$ \\
Power (\% max) & $5.7 \%$ & $P=0.03^{*}$ \\
Total time (min) & $11 \mathrm{~min}$ & $P=0.57$
\end{tabular}

${ }^{*} P<0.005$ statistically significant. this study. The patients were followed up for 6-month period.

All surgeries went uneventful; there were no cases of anterior radial capsule tear, posterior capsule tear, vitreous presentation, or loss. Although the nuclear hardness was of grade 3 or above (in a scale of 5), no eye suffered of thermal corneal burn. The amount of infusion solution was noticeably less than infusion fluid volume used during conventional coaxial phaco.

During the postoperative follow-up period, only three eyes suffered postoperative iritis of grade $2+$. Iritis rapidly improved within 1 week after the surgery on topical steroids four times per day for 1 week, followed by gradual tapering among the following 2 weeks.

The mean EPT was $4.3 \mathrm{~s}$. The average ultrasound used during the surgeries was $5.7 \%$. The mean total operation time was $11 \mathrm{~min}$ and $20 \mathrm{~s}$ (Table 2). This technique induced significantly less change in corneal astigmatism. At 3 weeks after the surgery, the amount of astigmatic shift compared to preoperative $\mathrm{K}$ readings was $0.34 \mathrm{D}$ with average axis rotation of $5.2^{\circ}$. No single case of

Table 2 Surgery parameters and postoperative complications

\begin{tabular}{|c|c|c|c|c|c|c|}
\hline No. & Age (years) & Sex & Degree of hardness & EPT (effective phacotime) & Duration of surgery & Postoperative complications \\
\hline 1 & 65 & M & Grade 3 & 3.3 & $9.0 \mathrm{~min}$ & - \\
\hline 2 & 60 & M & Grade 4 & 4.0 & $8.0 \mathrm{~min}-30 \mathrm{~s}$ & Grade $1+$ iritis \\
\hline 3 & 55 & $\mathrm{M}$ & Grade 3 & 3.3 & $11.0 \mathrm{~min}-46 \mathrm{~s}$ & - \\
\hline 4 & 63 & $\mathrm{M}$ & Grade 3 & 4.6 & $11.0 \mathrm{~min}-46 \mathrm{~s}$ & - \\
\hline 5 & 59 & $\mathrm{~F}$ & Grade 4 & 4.4 & $11.0 \mathrm{~min}-26 \mathrm{~s}$ & Grade $2+$ iritis \\
\hline 6 & 57 & $\mathrm{M}$ & Grade 5 & 4.0 & $11.0 \mathrm{~min}-39 \mathrm{~s}$ & Grade $1+$ iritis \\
\hline 7 & 70 & $\mathrm{M}$ & Grade 3 & 4.9 & $11.0 \mathrm{~min}-26 \mathrm{~s}$ & - \\
\hline 8 & 72 & M & Grade 5 & 5.0 & $11.0 \mathrm{~min}-31 \mathrm{~s}$ & Grade $1+$ iritis \\
\hline 9 & 71 & M & Grade 3 & 3.4 & $11.0 \mathrm{~min}-26 \mathrm{~s}$ & Grade $1+$ iritis \\
\hline 10 & 56 & $\mathrm{~F}$ & Grade 3 & 4.2 & $11.0 \mathrm{~min}-29 \mathrm{~s}$ & - \\
\hline 11 & 69 & M & Grade 3 & 4.3 & $11.0 \mathrm{~min}-46 \mathrm{~s}$ & - \\
\hline 12 & 56 & M & Grade 4 & 4.1 & $11.0 \mathrm{~min}-25 \mathrm{~s}$ & Grade $1+$ iritis \\
\hline 13 & 70 & $\mathrm{M}$ & Grade 3 & 4.1 & $11.0 \mathrm{~min}-59 \mathrm{~s}$ & - \\
\hline 14 & 71 & M & Grade 5 & 5.5 & $13.0 \mathrm{~min}-21 \mathrm{~s}$ & Grade $1+$ iritis \\
\hline 15 & 68 & $\mathrm{~F}$ & Grade 3 & 4.1 & $11.0 \mathrm{~min}$ & - \\
\hline 16 & 67 & M & Grade 3 & 3.5 & $11.0 \mathrm{~min}$ & - \\
\hline 17 & 73 & $\mathrm{M}$ & Grade 4 & 4.9 & $11.0 \mathrm{~min}-22 \mathrm{~s}$ & Grade $1+$ iritis \\
\hline 18 & 71 & M & Grade 3 & 4.3 & $11.0 \mathrm{~min}-26 \mathrm{~s}$ & - \\
\hline 19 & 61 & $\mathrm{M}$ & Grade 3 & 3.4 & $11.0 \mathrm{~min}-26 \mathrm{~s}$ & - \\
\hline 20 & 57 & M & Grade 5 & 4.7 & $14.0 \mathrm{~min}$ & Grade $2+$ iritis \\
\hline 21 & 64 & $\mathrm{~F}$ & Grade 4 & 4.1 & $12.0 \mathrm{~min}$ & - \\
\hline 22 & 66 & M & Grade 4 & 4.2 & $12.0 \mathrm{~min}$ & Grade $1+$ iritis \\
\hline 23 & 58 & $\mathrm{M}$ & Grade 3 & 3.6 & $11.0 \mathrm{~min} \min -56 \mathrm{~s}$ & - \\
\hline 24 & 63 & M & Grade 3 & 3.4 & $12.0 \mathrm{~min}-31 \mathrm{~s}$ & - \\
\hline 25 & 59 & $\mathrm{~F}$ & Grade 3 & 3.4 & $11.0 \mathrm{~min}-29 \mathrm{~s}$ & - \\
\hline 26 & 65 & $\mathrm{M}$ & Grade 4 & 5.9 & $13.0 \mathrm{~min}-55 \mathrm{~s}$ & Grade $1+$ iritis \\
\hline 27 & 65 & M & Grade 4 & 4.5 & $11.0 \mathrm{~min}-25 \mathrm{~s}$ & Grade $2+$ iritis \\
\hline 28 & 69 & M & Grade 3 & 3.4 & $11.0 \mathrm{~min}-56 \mathrm{~s}$ & - \\
\hline 29 & 72 & $\mathrm{~F}$ & Grade 3 & 4.8 & $11.0 \mathrm{~min}-39 \mathrm{~s}$ & - \\
\hline 30 & 73 & $\mathrm{~F}$ & Grade 4 & 5.0 & $11.0 \mathrm{~min}-44 \mathrm{~s}$ & Grade $1+$ iritis \\
\hline 31 & 59 & M & Grade 4 & 4.1 & $12.0 \mathrm{~min}-33 \mathrm{~s}$ & Grade $1+$ iritis \\
\hline 32 & 72 & $\mathrm{M}$ & Grade 4 & 5.4 & $12.0 \mathrm{~min} \min -54 \mathrm{~s}$ & Grade $1+$ iritis \\
\hline 33 & 70 & $\mathrm{~F}$ & Grade 3 & 4.1 & $13.0 \mathrm{~min}-32 \mathrm{~s}$ & - \\
\hline
\end{tabular}


endophthalmitis had been reported in this study (Tables 1 and 2).

\section{Discussion}

There are several advantages to the bimanual phacoemulsification method of cataract extraction. First, smaller incisions theoretically prevent high-pressure fluctuation within the eye during the surgery and prevent the loss of viscoelasticity, and hence provide a more stable anterior chamber during the procedure. ${ }^{4}$ Steps in cataract surgery such as capsulorrhexis become easier to perform and may reduce the risk of peripheral extension. ${ }^{5,6}$ Second, the small corneal incisions heal quickly, induce less postoperative astigmatism, and are theoretically associated with reduced risk of endophthalmitis. ${ }^{2,6,7}$ Third, capturing nuclear fragments using a separate irrigation and aspiration probe is much easier than using the single handpiece. By using the bimanual method, the irrigating handpiece can be kept in a fixed position, and the aspirating handpiece is free to reach the fragments, whereas with the single phaco handpiece, irrigation from the sleeve tends to push fragments away from the phaco tip, making aspiration more difficult. ${ }^{4}$ Fourth, the separate aspirating and irrigating handpiece ports can be interchanged, giving the surgeon a number of approaches to reach nuclear or cortical fragments subincisionally. ${ }^{6}$

Disadvantages to bimanual phacoemulsification, until recently, had outweighed the advantages listed and were a barrier to widespread adoption of the bimanual phacoemulsification technique by cataract surgeons. Disadvantages include some of the following points. First, there were no IOL that could fit through the microincisions used in bimanual phacoemulsification. As a result, incisions needed to be enlarged to implant the lens, which many surgeons thought defeated the purpose of ultra small incisional surgery and negated many of the advantages of bimanual phacoemulsification. Second, without the protective irrigation sleeve, there was concern that the bare phaco needle might overheat during phacoemulsification and cause corneal wound burns. Third, the small size of the irrigating probes limits the maximum irrigation that can be provided to the eye. ${ }^{8}$ As a result, when high vacuum is used or after occlusion of the phaco tip, the irrigation provided by the irrigating tip may not be sufficient to maintain a stable anterior chamber, which can make many steps of the surgery technically difficult to complete. ${ }^{8}$ Another disadvantage is increased tendency of wound leak; however, in our study we did not require any suture as the stab incisions could be sealed completely with careful stromal hydration and confirmed by negative Seidel test at the end of the surgery.
Also, the surgeon should be careful not to inject too much balanced salt solution during the hydrodissection/ hydrodelineation via an ultrasmall incision as this may increase possibility of capsule block syndrome, especially with the use of high-density ocular viscoelastic devices. In our study, we have not met any case of capsule block syndrome owing to careful hydrodissection and delineation using 25-G cannula attached to $1 \mathrm{~cm}^{3}$ syringe.

The safety of bimanual microincision phacoemulsification using WhiteStar technology in dense nuclear sclerotic cataracts was further substantiated by a recent study performed by Olson. ${ }^{6}$ In this study, 18 consecutive patients with 3 or $4+$ nuclear sclerotic cataracts underwent 21-G bimanual phacoemulsification. No complications occurred during the procedure. On the first postoperative day, $72 \%$ of patients had no corneal oedema, and the mean level of anterior chamber inflammation for all patients was quite low. Olson $e t a l^{9}$ have also performed wound studies of cadaver eyes undergoing phacoemulsification with both the Sovereign with WhiteStar and the Alcon Legacy with AdvanTec (Alcon Laboratories, Fort Worth, TX, USA), and found less increase in wound temperatures with the Sovereign machine. Our study results are comparable to other studies carried out on rollable ThinOptX IOL. ${ }^{10,11}$

\section{Conclusions}

Cataract surgery has undergone a number of transformations in the last 10-15 years. The trend has been to minimize damage to the cornea by reducing incision sizes, developing new power modulations to prevent injury to the corneal endothelium, and creating foldable lenses that can fit through small incisions. Bimanual phacoemulsification appears to be the next step in the development of cataract surgery. Clinical studies have been positive, emphasizing the safety and efficacy of bimanual microincisional phacoemulsification on any of the currently available phaco systems and its potential as a minimally invasive cataract surgery. Future directions for cataract surgery and microincision cataract surgery (MICS) may include refractive lens exchange-a type of refractive surgery in which the crystalline lens is removed before a cataract forms to correct refractive error. Fine $e t a l^{8}$ recently described their technique for refractive lens exchange using MICS. They note that one of the main advantages to MICS is the improvement in surgical control throughout the procedure, which is the key when operating on myopes to minimize the risk of retinal detachment. ${ }^{8}$ It is clear that MICS is quickly becoming a procedure of interest for a number of ophthalmologists, and it can be expected to become even more common once smaller-incision IOLs become readily available. 


\section{References}

1 Linebarger EJ, Hardten DR, Shah GK, Lindstrom RL. Phacoemulsification and modern cataract surgery. Surv Ophthalmol 1999; 44: 123-147.

2 Shearing SP, Relyea RL, Louiza A, Shearing RL. Routine phacoemulsification through a one-millimetre non-sutured incision. Cataract 1985; 2: 6-11.

3 Olson RJ. Early experience with microphaco. Clin Surg Ophthalmol 2004; 22: 86-91.

4 Soscia W, Howard JG, Olson RJ. Bimanual phacoemulsification through 2 stab incisions: a wound temperature study. J Cataract Refract Surg 2002; 28: 1039-1043.

5 Fine IH, Packer M, Hoffman RS. New phacoemulsification technologies. J Cataract Refract Surg 2002; 28: 1054-1060.

6 Fine IH, Packer M, Hoffman RS. Power modulations in new phacoemulsification technology: improved outcomes. J Cataract Refract Surg 2004; 30: 1014-1019.
7 Olson RJ. Clinical experience with 21-gauge manual microphacoemulsification using Sovereign WhiteStar Technology in eyes with dense cataract. J Cataract Refract Surg 2004; 30: 168-172.

8 Fine IH, Hoffman RS, Packer M. Optimizing refractive lens exchange with bimanual microincision phacoemulsification. J Cataract Refract Surg 2004; 30: 550-554.

9 Olson RJ, Jin Y, Kefalopoulos G, Brinton J. Legacy AdvanTec and Sovereign WhiteStar: a wound temperature study. J Cataract Refract Surg 2004; 30: 1109-1113.

10 Alio JL, Schimchak P, Montes-Mico R, Galal A. Retinal image quality after microincision intraocular lens implantation. J Cataract Refract Surg 2005; 31(8): 1557-1560.

11 Pandey SK, Werner L, Agarwal A, Agarwal A, Lal V, Patel N et al. Phakonit. Cataract removal through a sub- $1.0 \mathrm{~mm}$ incision and implantation of the ThinOptX rollable intraocular lens. J Cataract Refract Surg 2002; 28(9): 1710-1713. 